\title{
REPRESENTACIONES SOCIALES SOBRE GÉNERO EN ACTORES RELEVANTES VINCULADAS CON LA DESPENALIZACIÓN DE LA INTERRUPCIÓN DEL EMBARAZO EN CHILE*
}

\author{
SOCIAL REPRESENTATIONS ON GENDER IN RELEVANT ACTORS \\ LINKED WITH THE DEPENALIZATION OF THE INTERRUPTION \\ OF PREGNANCY IN CHILE
}

\author{
MIRLIANA RAMÍREZ-PEREIRA**, ADELA MONTERO VEGA***
}

Resumen: En 2017, Chile despenalizó la interrupción voluntaria del embarazo (IVE) por tres motivos específicos, evidenciándose en el debate público y legislativo representaciones sociales relacionadas con el género. El objetivo de este artículo es describir y analizar las representaciones sociales sobre género en actores relevantes durante la discusión sobre la objeción de conciencia y despenalización de la IVE en Chile. La metodología utilizada fue de tipo cualitativa. Se realizaron 42 entrevistas semiestructuradas a actores relevantes. Posterior al análisis de contenido y triangulación de la información, emergieron las siguientes subcategorías: "Mujer - madre", "Mujer - sujeto de derechos", "Mujer - vulnerable", "Mujer - discriminada", "Mujer - no confiable" y "Derechos de la mujer - derechos del feto". Como conclusión, resulta esencial abolir los estereotipos negativos que afectan a la mujer, reconociéndola como persona titular de derechos. Se propone promover la investigación en torno al aborto, que permita comprender el fenómeno considerando el género, instaurando políticas públicas que potencien el empoderamiento y reconocimiento de los derechos de las mujeres.

Palabras clave: Actitud frente al aborto en tres causales, investigación cualitativa, género, salud reproductiva, representaciones sociales.

\footnotetext{
* Este artículo deriva del Proyecto de Investigación "Representaciones Discursivas sobre Derechos y Deberes Profesionales, Institucionales y Rol del Estado, relacionadas con la Objeción de Conciencia Sanitaria y Despenalización de la Interrupción Voluntaria del Embarazo, por tres causales en Chile", financiado por el Fondo Nacional de Desarrollo Científico y Tecnológico (Fondecyt), del Gobierno de Chile, entre los años 2016 y 2019 (Grant № 1160602). La investigación fue aprobada por el Comité de Ética en Investigación en Seres Humanos de la Facultad de Medicina, Universidad de Chile (009/2016). Agradecemos al Fondo Nacional de Desarrollo Científico y Tecnológico, a todas las personas que hicieron posible este proyecto, en especial a quienes participaron en las entrevistas y a las mujeres chilenas.

** Doctora en Enfermería. Académica del Departamento de Enfermería, Facultad de Medicina, Universidad de Chile, Santiago, Chile. Correo electrónico: mirliana@uchile.cl. Orcid: https://orcid. org/0000-0003-1439-4162

*** Magíster en Bioética. Académica del Centro de Medicina Reproductiva y Desarrollo Integral del Adolescente, Facultad de Medicina, y del Centro de Estudios de Ética Aplicada, Facultad de Filosofía y Humanidades, Universidad de Chile, Santiago, Chile. Correo electrónico: amontero@uchile.cl. Orcid: https://orcid.org/0000-0003-1811-8309
} 
Aвstract: In 2017, Chile decriminalized the voluntary interruption of pregnancy (VIP) for three specific reasons, evidencing gender-related social representations in the public and legislative debate. The aim of this article is to describe and analyze social representations about gender in relevant actors during the discussion on conscientious objection and decriminalization of VIP. The methodology used was qualitative. Fortytwo semi-structured interviews were conducted with relevant actors. After content analysis and triangulation of the information, the following subcategories emerged: "Woman - mother", "Woman - subject of rights", "Woman - vulnerable", "Woman - discriminated", "Woman - untrustworthy" and "Rights of women - rights of the fetus". In conclusion, it is essential to abolish negative stereotypes towards women, recognizing them as rights holders. It is proposed to promote research on abortion that would allow to understand the phenomenon considering gender, establishing public policies that enhance the empowerment and recognition of women's rights.

KEYwORDs: Position regarding abortion in three specific reasons, qualitative research, gender, reproductive health, social representations.

Recibido: 21.12.2019. Aceptado: 28.07.2020.

\section{INTRODUCCIÓN}

$\mathrm{D}$ ESDE QUE EN SEPTIEMBRE de 1989 se derogó el aborto terapéutico, transcurrieron 28 años para que Chile lograra despenalizar la interrupción voluntaria del embarazo (IVE), al menos en tres motivos específicos, dando origen a la Ley 21.030: que la mujer se encuentre en riesgo vital, de modo que la interrupción del embarazo evite un peligro para su vida; que el embrión o feto padezca una patología congénita adquirida o genética, incompatible con la vida extrauterina independiente, de carácter letal; y que el embarazo haya resultado de una violación (Gobierno de Chile, 2017).

Desde el retorno de la democracia, acontecido en 1990, hubo múltiples instancias parlamentarias que resultaron infructuosas para incorporar el aborto en la legislación. Durante la discusión de la ley 21.030, e incluso antes de que el Gobierno de esa época enviara la moción parlamentaria (Montero et al., 2017), hubo una fuerte resistencia de grupos conservadores, con una polarización de las posiciones, especialmente en torno a la objeción de conciencia ${ }^{1}$, que apareció como tema central y se mantuvo durante todo el período de tramitación legislativa, que culminó con la sentencia del Tribu-

\footnotetext{
${ }^{1}$ Según Casas y Dides (2007), la objeción de conciencia "supondría la regulación de la exención de cumplimiento de una obligación jurídica fundamental, derivada normalmente de las relaciones laborales o funcionarias (contrato de trabajo o estatuto funcionario); está dirigida a los poderes públicos y a los particulares y siempre se plantea frente a una obligación personal. El accionar del objetor responde únicamente a dejar a salvo su conciencia, ya sea por razones religiosas o filosóficas" (p. 202).
} 
nal Constitucional chileno (Montero \& Villarroel, 2018). Este organismo reconoció la constitucionalidad de la IVE en estas causales, pero basándose en la libertad de conciencia y en la libertad de asociación y autonomía de los cuerpos intermedios, amplió la objeción de conciencia a los equipos de salud que participen de manera directa o indirecta en la interrupción del embarazo al interior del pabellón quirúrgico y permitió no realizar las prestaciones a los establecimientos privados, aun cuando reciban financiamiento estatal (Tribunal Constitucional, 2018).

Durante la discusión y tramitación de la ley 21.030, participaron numerosos actores sociales provenientes de diversos sectores: legislativo, académico, gubernamental, religioso y sociedad civil, entre otros, quienes desde sus propias creencias y voces que debían representar, decidieron sobre los derechos sexuales y reproductivos de las mujeres. Cabe señalar que la actuación de grupos de mujeres, pertenecientes a la sociedad civil organizada, constituyó un fuerte respaldo e impulso para que se lograra la aprobación de la ley (Fernández, 2018).

El impacto que representó en nuestra sociedad la discusión sobre el aborto en este período histórico nos motivó a realizar una investigación destinada a explorar los discursos de actores relevantes, principalmente en torno a la objeción de conciencia. Sin embargo, en el marco de esta investigación cualitativa, surgió de manera emergente, el discurso relacionado con las representaciones sociales de género. Por ello, el propósito de este artículo es describir y analizar estas representaciones sociales que surgieron en actores clave, durante el proceso que permitió despenalizar el aborto en Chile.

\section{METODOLOGÍA}

Siguiendo la línea de la investigación principal, realizamos un estudio cualitativo, bajo el paradigma pospositivista, el cual, según Lincoln et al. (2017), apunta hacia la objetividad que se convierte en un ente regulador, sin constituir una meta en sí misma (Johnson \& Rasulova, 2017). Por consiguiente, la presentación de los resultados se realiza intentando mantener un resguardo de objetividad.

\section{Participantes}

Para la selección de informantes clave, se realizó en primera instancia una búsqueda y revisión de documentos con el propósito de identificar y seleccionar la información relevante, presente en la discusión nacional y 
pública, que permitiera el abordaje inicial y el análisis crítico de los diferentes enfoques sobre la objeción de conciencia y su relación con la IVE. Para ello, se accedió a los reportes de los principales medios de comunicación de distribución nacional, que contaran con sitio web y buscadores internos, que permitieran identificar las principales "piezas informativas" (columnas de opinión, editoriales, crónicas, entrevistas, reportajes, comunicados de prensa, entre otras), relacionadas con la objeción de conciencia y aborto (Montero et al., 2017).

Los resultados de esta búsqueda fueron sistematizados en una base de datos, donde además de consignar la fuente, la fecha de emisión y el tipo de pieza informativa, el contenido de cada una de ellas fue desagregado según las/los informantes clave mencionadas/os y la forma en cómo hacían alusión a la objeción de conciencia, al aborto en general y al aborto en tres causales. De esta forma se consignó como 'caso' los datos provenientes de cada actor mencionado en las piezas de prensa y las alusiones que hacía a las temáticas señaladas. Esto nos permitió identificar un total de 251 piezas informativas en once medios de comunicación, correspondiente al discurso de 215 actores que participaron activamente en el debate sobre objeción de conciencia y aborto en Chile (Montero et al., 2017).

Paralelamente, se realizó consulta y seguimiento periódico de la documentación del Congreso de la República, especialmente relacionada con la tramitación del proyecto que dio origen a la Ley 21.030. A través de la página web institucional del Congreso, se tuvo acceso a los tres informes de las sesiones de la Comisión de Salud, de la Comisión de Constitución, Legislación, Justicia y Reglamento, y de la Comisión de Hacienda de la Cámara de Diputados; a los siete informes de las comisiones similares correspondientes al Senado de la República y a las sesiones plenarias de la Cámara y del Senado. Esto permitió identificar a quienes, por la preponderancia de su discurso en torno a la objeción de conciencia y aborto, fueron relevantes.

Con esta información se confeccionó una matriz de actores, seleccionando a quienes habían tenido relevancia en ambos espacios (prensa y Congreso), lo que permitió realizar un muestreo teórico o propositivo, no probabilístico, seleccionando como unidades de análisis un total de 64 informantes clave, considerando como criterio de inclusión que hubiesen estado directamente involucrados en la discusión de la Ley 21.030, independientemente de su postura personal frente al aborto.

Las/os informantes clave identificadas/os que accedieron a participar, recibieron una invitación vía correo electrónico, donde se les informaba del estudio. Los datos de la entrevista fueron recopilados únicamente por 
integrantes del equipo de investigadores desde junio de 2017 hasta julio de 2018. Incluyeron a: autoridades y asesores ministeriales de la segunda administración de la Presidenta Bachelet; autoridades, académicos y especialistas de universidades e instituciones de salud públicas y privadas; miembros del Tribunal Constitucional; integrantes de la Comisión de Salud y de la Comisión de Constitución, Legislación, Justicia y Reglamento de la Cámara y el Senado; expertos en Bioética; líderes de estudiantes universitarios; representantes de la Iglesia Católica y Evangélica; miembros de asociaciones profesionales y de funcionarios de la salud y representantes de la sociedad civil, cuyos nombres y afiliación han sido retenidos para proteger la confidencialidad de su identidad.

Por la riqueza de la información aportada, se alcanzó la saturación teórica con un total de cuarenta y dos actores. Cuarenta participantes proporcionaron antecedentes sociodemográficos en forma anónima. El promedio de edad fue de 51,3 años (23 - 78 años). El 52,5\% fueron mujeres. El 50\% corresponde a abogados/as: $35 \%$ médicos/as y $15 \%$ corresponde a otras profesiones (trabajador/a social, matrón/a, teólogo/a, antropólogo/a, técnico/a en computación, dirigente/a universitario/a). Respecto de la principal corriente de pensamiento, treinta y nueve participantes contestaron esta dimensión. El 53,8\% se considera "liberal"; 17,9\% "medianamente liberal"; $15,4 \%$ se considera "ni liberal ni conservador"; $10,3 \%$ "medianamente conservador" y solo 2,6\% se cataloga como "conservador". En relación con las creencias, $48,7 \%$ se considera "sin creencias"; $38,5 \%$ se considera "católico"; $7,7 \%$ "cristiano o evangélico"; un 2,6 \% declara pertencer a la religión judía y un $2,6 \%$ se declara francamente "ateo".

\section{Instrumento de recolección de la información}

Como técnica de recolección de información se usó la entrevista semiestructurada. Esta técnica es utilizada frecuentemente en investigación cualitativa y se comprende como un diálogo entre investigador y participante, guiado por un protocolo de entrevista flexible y complementado por preguntas de seguimiento, sondeos y comentarios (Dejonckheere \& Vaughn, 2019).

La pauta de entrevista fue construida en base a la revisión de la literatura y piloteada antes de ser aplicada, con la participación de 2 personas de características similares a los participantes. Las observaciones realizadas fueron incorporadas a la pauta definitiva. La entrevista tuvo dos secciones, la primera parte incluyó una encuesta anónima de antecedentes sociodemo- 
gráficos. En la segunda sección se realizaron preguntas abiertas centradas en los elementos de las representaciones sociales relacionadas principalmente con el aborto y la objeción de conciencia.

La entrevista semiestructurada introdujo a las principales áreas temáticas de investigación, permitiendo a las/los participantes determinar la profundidad y dirección de sus respuestas, con una duración promedio de 56.7 minutos. Se obtuvo el consentimiento informado antes de cada entrevista.

Las entrevistas fueron transcritas por dos asistentes, quienes se comprometieron a la confidencialidad de la información. Antes del análisis, las/los participantes tuvieron la oportunidad de revisar y/o editar su entrevista. Los archivos de las transcripciones se encriptaron con un código. Se informó a las/los participantes que podían dar por finalizada su participación en cualquier momento sin mediar explicación, teniendo la libertad de negarse a responder preguntas específicas.

\section{Análisis de los datos}

Se realizó según análisis de contenido, siguiendo los pasos descritos a continuación: en primer lugar, se leyeron las entrevistas transcritas con el fin de obtener una idea global y se elaboraron unidades de significado, las que luego fueron etiquetadas, formulando códigos y agrupando estos códigos en categorías (Erlingsson \& Brysiewicz, 2017).

En esta investigación, el proceso de análisis se trianguló con la participación de ambas autoras. Se utilizó un proceso inductivo mediante codificación abierta. En la formulación de las categorías, los códigos se compararon en términos de similitudes y diferencias. Los códigos con significados similares se asignaron a una categoría. En la siguiente etapa, las categorías iniciales se compararon e integraron, logrando una categoría común. En la etapa de abstracción, las autoras, finalmente, etiquetaron las categorías y subcategorías de acuerdo con los códigos y sus contenidos, obteniendo así el contenido de la categoría. Adicionalmente, el análisis se apoyó con el software ATLAS ti ${ }^{\text {th }} 8.0$.

Se realizó triangulación de datos entre investigadores, revisión y edición de la entrevista por las/los participantes previo al análisis, tiempo extenso de recolección de datos. Además, se presentaron los resultados en un seminario abierto, recogiendo sugerencias de las/os asistentes, representadas/ os por varias personas participantes del estudio y otras provenientes de la sociedad civil y de la academia. 


\section{RESULTADOS}

Como se mencionó, durante el análisis emergió con fuerza la perspectiva de género en el discurso de las/los participantes, identificando 6 categorías: "Mujer - madre"; "Mujer - sujeto de derechos"; "Mujer - vulnerable", "Mujer - discriminada", "Mujer - no confiable" y "Derechos de la mujer - derechos del feto".

Estas categorías se encuentran determinadas principalmente por el discurso del/la participante en torno a la mujer. Este discurso en ocasiones es presentado por el/la participante en $1^{\text {a }}$ persona, aludiendo a la propia concepción ideológica sobre el tema. Sin embargo, en otras circunstancias, elabora su contenido en $3^{\mathrm{a}}$ persona, refiriéndose al discurso que estuvo igualmente presente en la discusión de la ley 21.030.

\section{Categorías}

\section{Mujer - madre:}

Para participantes que se autodefinen como ideológicamente conservadores, la función principal de la mujer es la reproducción, asociando su vida a la maternidad, como un bien superior y aporte indiscutible a la sociedad. Se refieren a ella como la madre y no como mujeres. La maternidad es comprendida como un deber y al mismo tiempo una bendición, negando de este modo la posibilidad de que la mujer elija un camino distinto al que la sociedad determinó para ella, absolutamente ajustado a su género.

Desde el minuto en que tienes que hacer el aborto a escondidas, inevitablemente produce esa sensación, del estereotipo tradicional de la mujer, como madre, que una mujer no quiera ser madre..., como que algo malo debe tener esa mujer si no quiere tener hijos. (Abogada, ONG internacional)

El discurso principal, relevante en la discusión legislativa, fue el rol de las mujeres y el mandato social de la maternidad, de raigambre religiosa... Entonces ahí tú tienes súper claro, esto era lo que veníamos todo el rato escuchando por debajo. Finalmente, lo que está en juego es el mandato de maternidad de las mujeres y las creencias religiosas que imponen a la mujer ese rol en la sociedad. (Abogada, representante sociedad civil)

2. Mujer - sujeto de derechos

Para quienes apoyan la interrupción voluntaria del embarazo, la mujer se representa como sujeto de derechos, dentro de los cuales el respeto de los 
derechos sexuales y derechos reproductivos constituye un pilar en la lucha por la equidad de género. Desde esta mirada, la mujer deja de ser un sujeto pasivo, receptora de políticas públicas, convirtiéndose en una persona que toma partido como ciudadana en aquellas acciones que la empoderan e impulsan hacia mejoras en su calidad de vida.

Desde el punto de vista de la jueza, de los jueces, nuestro interés en la despenalización del aborto es porque la realidad, es un delito, que todos los tratados internacionales y todas las normas internacionales señalan como una cuestión que tiene que ser discutida en un Estado, que tiene que ser resuelto democráticamente y que está muy vinculado al respeto a los derechos humanos de las mujeres en especial y eso implica los derechos sexuales y reproductivos. (Magistrada)

3. Mujer - vulnerable

En el discurso de los participantes, emerge la idea de que la mujer cuando aborta lo hace presionada u obligada por su contexto social, familiar o cultural, y no por una decisión propia, lo que la sitúa en una extrema vulnerabilidad para tomar la decisión de interrumpir el embarazo.

En el acoger, en el proteger a estas mujeres en un momento de vulnerabilidad, donde además la sociedad, después de todas sus intervenciones, además, las va a señalar, o sea, me parece que es agredir a quien necesita, justamente, ser protegido, acogido. (Representante colegio profesional 2)

Yo no lo sé, pero sí a mí me llamó mucho la atención que ese fuera el argumento de muchas autoridades, "pobre mujer", o sea... estamos haciendo esto por ella, para que ella no se vaya a sentir culpable después. Cuando el problema yo creo que es otro, es que nosotras, nosotros culpabilizamos a las mujeres que se realizan abortos. (Dirigente estudiantes universitarios)

\section{Mujer - discriminada}

Quienes tienen una posición a favor del aborto refieren que las mujeres son discriminadas por el sector privado de salud, específicamente por las instituciones de salud previsional (Isapres), al otorgarles un seguro en la medida en que paguen un valor más alto que los hombres de la misma edad, debido a que conforman una población de mayor riesgo frente a la posibilidad de un embarazo. 
También algunas mujeres poseen planes de seguro de salud exclusivos para recibir atención en determinados establecimientos privados, los que, al no proporcionar la prestación respecto de la IVE por declararse objetores, dificultarían el acceso a estas prestaciones.

Creo que las mujeres que son beneficiarias de esas Isapres, sobre todo de esos planes cerrados..., esto nadie lo planifica, al momento de suscribir a un plan de salud, que ya tiene muchos vicios porque son cerrados, esas mujeres igual se van a enfrentar a la discriminación de su centro de salud, ya estando en una posición muy vulnerable y me parece que... no es entender nada del rol sanitario. (Representante colegio profesional 2)

Yo miro con desconfianza la objeción de conciencia. Y como la objeción de conciencia solo funciona prácticamente en materia de derechos sexuales y reproductivos, me da la sensación de que hay temas de género detrás. Porque las mismas razones podrían ocuparse en otros contextos y respecto de otras personas (no mujeres) y a nadie se le ocurre ocuparla en otros contextos, para otros sujetos. (Abogada, profesora derecho constitucional 2)

5. Mujer - no confiable

Otra categoría que emerge desde las/los participantes se relaciona con el cuestionamiento que realizan estos/as sobre la confiabilidad del relato de las mujeres que solicitan la interrupción, fundamentalmente en la causal violación. De acuerdo con los/as participantes, existe la posibilidad de que la mujer proporcione información no fidedigna respecto de la violación, con el objetivo de acceder al aborto frente a un embarazo no deseado, infringiendo de esta forma el espíritu de la ley.

Ya no es una paciente, es una potencial delincuente, es una mujer sospechosa y los que intervienen y toman decisiones ya no son los médicos, son los fiscales, son los jueces, los policías. (Abogada, representante sociedad civil)

Y por otro lado respecto a una usuaria que pudiera invocar esa causal, porque al final se ha hecho tal..., caricatura del tema que, casi que la usuaria va a llegar a pedir esta atención y la van a mirar con desconfianza y van a mirar si es que estaba golpeada o si se veía triste, o si parecía mentirosa o si cualquier cosa... (Representante profesionales universitarios) 


\section{Derechos de la mujer - derechos del feto}

Existe un no reconocimiento del derecho de la mujer a decidir, en el que prima el derecho a la vida del feto al ser considerado persona y miembro de la especie humana, con esto adquiere un estatus moral igual o superior a los derechos de la mujer.

Es la idea de que el feto, o sea, el valor de la vida está por sobre cualquiera y no hay una valoración de los derechos de la mujer, o sea, yo siento que de fondo es un discurso conservador, es un no reconocimiento de los derechos de la mujer. (Profesional médico, bioeticista)

Creo que este es un gran retroceso en la humanidad en Chile, como valores humanos para todos. A mí me parece que esto de que no se respete la vida de un niño en gestación es algo sorprendente, es, incluso, si uno analiza la ley que dice: "protege la vida del que está por nacer". Yo no entiendo en qué parte esto no es una vida de un ser humano que está por nacer. (Profesional médico, institución con ideario)

\section{DISCUSIÓN}

Las representaciones sociales forman parte de la vida cotidiana y del sentido común de las sociedades. Son constituidas y constituyentes de creencias, valores y experiencias, de esta forma, las personas se vinculan y forman redes, fortaleciendo su sentido de identidad y pertenencia (Marková, 2017). También son comprendidas como conjuntos dinámicos cuya característica es producir comportamientos y relaciones. En este sentido, son formas de pensamiento constituido, debido a que conforman productos socioculturales que intervienen en la vida social como estructuras preformadas que sirven como marco de interpretación; y al mismo tiempo formas de pensamiento constituyente, en el sentido de que intervienen en la elaboración o conformación del objeto mismo que representan, contribuyendo a configurar la realidad social de la que forman parte y determinando en diversa medida sus efectos en la vida cotidiana (Mora, 2002).

El género es una construcción social potenciada por la cultura, política y prácticas sociales que definen los roles de mujeres, niñas, hombres y niños, como así también, lo que significa lo masculino y lo femenino. Los roles de género son enseñados, aprendidos e interiorizados y varían entre y dentro de las culturas. El género define los deberes y responsabilidades esperadas de mujeres, niñas, hombres y niños en cualquier momento de sus 
vidas y establece barreras, oportunidades y privilegios que puedan disfrutar a lo largo de sus vidas (IASC, 2017). El género también es un determinante de la salud, ya que estructura las oportunidades y los riesgos en el ciclo de vida. Mujeres y hombres no solo son diferentes biológicamente, sino que también difieren en los roles y responsabilidades asignados por la sociedad (Matud, 2016).

En la esfera pública y desde los discursos de actores clave en nuestro país, es posible dilucidar las categorías del análisis de contenido que permiten comprender cuáles son las representaciones sociales sobre la mujer en las que se configuró la actual legislación y que ya estaban presentes de manera explícita durante la elaboración de la actual Constitución Política. Así, en las Actas Oficiales de la Comisión Constituyente (Gobierno de Chile, 1974), se enarbolaron comentarios vinculados con la responsabilidad de la mujer frente al embarazo, que también se condice con el discurso de las/ os participantes contrarias/os a la IVE. En estas Actas, destaca el hecho de que la madre (no se hace referencia a la mujer), debe tener al hijo en cualquier circunstancia, aunque posea malformaciones, sea producto de una violación, no sea deseado o incluso si puede provocarle la muerte. También el aborto fue homologado con un homicidio, estando la mujer obligada indefectiblemente a continuar el embarazo (Gobierno de Chile, 1974).

Según Garrido-Luque et al. (2018), existe una correspondencia entre ser mujer y ser madre y sigue primando el aspecto biológico de la valoración por sobre la noción de género como construcción social. Sus hallazgos se corresponden con los observados en este estudio, según el cual, el ala conservadora de los actores clave en la discusión de la ley, ha explicitado el deber de la mujer de ser madre y responder a los mandatos de la sociedad.

Según otra autora, la mujer es vista como el pilar fundamental de la familia, debiendo concentrar la afectividad y la responsabilidad de mantener el núcleo familiar, asumiendo las consecuencias de la disfuncionalidad, con el fracaso o éxito que se pueden dar dentro de estas relaciones, fortaleciendo así la idea de la obligación de ser una "buena esposa y madre" (Santos et al., 2018, p. 774). Este postulado reafirma nuestros hallazgos en los discursos de las/los participantes y nos permite señalar que la representación social sobre el proyecto de vida de la mujer, pudo influir en el discurso de los grupos conservadores que se oponían a la despenalización del aborto.

En nuestra investigación emerge la categoría "mujer - vulnerable", que debe ser protegida y apoyada en la decisión de abortar, a razón de que el aborto es algo que ella realmente no desea, pero a lo que se ve obligada por presión del entorno. Esta categoría se vincula con el ejercicio de los derechos sexuales y reproductivos por parte de la mujer, debido a que, si 
los legisladores y quienes toman las decisiones resuelven según estos preceptos, podrían eventualmente vulnerar el derecho a decidir de la mujer, obligándola a una maternidad forzada y a un proceso de acompañamiento no deseado. Mendoza (2015) plantea que, en las representaciones sociales como objeto de estudio, la esencia de cada sexo emerge desde lo biológico, y según esto se distingue lo que es propio de la mujer o atribuido a la mujer, con una serie de cualidades típicas, como son la fragilidad y la emotividad.

De acuerdo con Duarte Cruz y García-Horta (2016), el género impacta la clase, la raza y la posición social, sin embargo, también impacta sobre las concepciones de justicia entre hombres y mujeres. Por ello, la administración de la justicia debe promover e incorporar los derechos humanos con perspectiva de género en las políticas públicas y sociales, nacionales e internacionales.

Los antecedentes expuestos en el párrafo anterior se condicen con la categoría "Mujer - sujeto de derecho". Quienes apoyan la IVE, consideran que las mujeres deben ejercer sus derechos sexuales y reproductivos para poder decidir sobre el aborto. Asimismo, advierten sobre una eventual colisión de derechos, entre los del feto y los de la mujer. Por el contrario, para los grupos conservadores, opositores al aborto, existe la percepción de igualdad de derechos, apareciendo en algunas oportunidades la idea de que los derechos del no nacido son de rango igual e incluso superior a los derechos de la mujer.

Bivort y Martínez-Labrín (2016) refiere que en su investigación emerge como tema relevante la percepción de discriminación respecto del acceso igualitario a la salud previsional, lo que estaría relacionado con el sistema económico neoliberal que permea todos los niveles y espacios del país. Desde la mirada neoliberal la salud se convierte en un bien de consumo y, según esta, el acceso está determinado por la capacidad de pago de las personas. En el caso de mujeres en edad fértil, con una alta posibilidad de embarazo, las instituciones privadas de salud las castigan con un precio mayor del seguro, produciéndose una suerte de discriminación. Esta discriminación también emerge en la presente investigación; cuando las/los participantes manifiestan que las instituciones de salud privadas tienen como objetivo el lucro, excluyendo con precios discriminatorios y planes exclusivos ligados a determinados grupos económicos a las mujeres en edad fértil.

Igualmente, es importante relevar la categoría "Mujer - no confiable", la que aparece en las/los participantes contrarias/os a la IVE, quienes consideran a la mujer capaz de alterar su relato con el fin de acceder a la interrupción. Si legisladores y otros actores clave parten de la base de que las mujeres faltan a la verdad, estas difícilmente podrán acceder a una adecuada 
atención y cuidado en circunstancias extremas, como es el embarazo como consecuencia de la violencia sexual (Tuerkheimer, 2017).

\section{CONCLUSIONES}

Como se desprende de nuestros hallazgos, aun cuando hay quienes reconocen a la mujer como titular de derechos, en la sociedad se identifica una imagen estereotipada, vinculada con un rol como reproductora, siendo considerada vulnerable, incapaz de tomar decisiones respecto de su sexualidad y su cuerpo. Existe además una noción negativa que desconfía del relato de la mujer, banalizando su condición de persona (Montero y Ramírez-Pereira, 2020).

Nuestro estudio confirma lo que muestra la historia de la humanidad. A pesar de estar en pleno siglo XXI, sigue presentándose una asociación entre el hecho de ser mujer y el de poseer menos derechos legales que los hombres. En otras palabras, en una sociedad donde existe una fuerte argumentación basada en el acceso al conocimiento y digitalización, en que se discuten los derechos humanos de quinta y sexta generación, incluyendo a las máquinas y organismos tecnológicos y genéticamente modificados, los representantes de los poderes del Estado continúan tomando decisiones según representaciones sociales presentes hace siglos en la humanidad, manteniendo relegadas a la mujeres en una posición desmedrada, como ciudadanas de segunda clase, con limitaciones para decidir sobre su propio cuerpo, su salud y su vida.

Según lo anterior, es necesario promover la investigación en ciencias sociales y humanidades que contemplen la dimensión de la salud, en especial respecto del acceso al aborto, considerando la perspectiva de género que permita comprender el fenómeno para instaurar políticas públicas y estrategias que refuercen el empoderamiento y reconocimiento de los derechos de las mujeres.

\section{REFERENCIAS}

Bivort, B. y Martínez-Labrín, S. (2016). Mujeres jóvenes y ciudadanía. Revista de Sociología e Política 24(59), 25-37.

Casas Becerra, L. y Dides Castillo, C. (2007). Objeción de conciencia y salud reproductiva en Chile: Dos casos paradigmáticos. Acta Bioethica 13(2), 199-206. Doi:10.4067/s1726-569x2007000200007. 
Dejonckheere, M. \& Vaughn, L. M. (2019). Semistructured interviewing in primary care research: a balance of relationship and rigour. Journal of Family Medicine and Health Care 7, e000057. Doi:10.1136/fmch-2018-000057. Disponible en: https://fmch.bmj.com/content/7/2/e000057

Duarte Cruz, J. M. y García-Horta, J. B. (2016). Igualdad, Equidad de Género y Feminismo, una mirada histórica a la conquista de los derechos de las mujeres. Revista CS 18, 107-158. Disponible en: http://www.scielo.org.co/ $\mathrm{pdf} / \mathrm{recs} / \mathrm{n} 18 / \mathrm{n} 18 \mathrm{a} 06 . \mathrm{pdf}$

Erlingsson, C. \& Brysiewicz, P. (2017). A hands-on guide to doing content analysis. African Journal of Emergency Medicine 7(3), 93-99. Disponible en: https://www.sciencedirect.com/science/article/pii/S2211419X17300423

Fernández, M. (2018). Retrospectiva de la sociedad civil organizada en Chile: 25 años de ACCIÓN. Disponible en: https://fdocuments.mx/document/retrospectiva-de-la-sociedad-civil-organizada-en-chile-25-25-aos-de-accin. html

Garrido-Luque, A., Álvaro-Estramiana, J. L., y Rosas-Torres, A. R. (2018). Estereotipos de género, maternidad y empleo: un análisis psicológico. Pensando Psicología 14(23), 1-14. Doi: https://doi.org/10.16925/pe.v14i23.2261

Gobierno de Chile (1974). Actas Oficiales Comisión Constituyente. Santiago, Chile [en línea]. Santiago, Chile. Disponible en: https://www.bcn.cl/obtie nearchivo?id=recursoslegales/10221.3/3767/2/Tomo_III_Comision_Ortuzar.pdf

Gobierno de Chile (2017). Ley 21030 Regula la Despenalización de la Interrupción Voluntaria del Embarazo en tres Causales, Diario oficial del gobierno de Chile. Disponible en: http://www.diariooficial.interior.gob.cl/publicaciones/2017/09/23/41866/01/12762 48.pdf [2019, 25 de septiembre]

Inter-Agency Standing Committee (IASC). (2017). Why gender equality is essential to humanitarian action. IASC, The Gender Handbook for Humanitarian Action (pp 15-26). Ginebra: IASC.

Johnson, S. \& Rasulova, S. (2017). Qualitative impact evaluation: incorporating authenticity into the assessment of rigour. Journal of Development Effectiveness 9(2), 263-276.

Lincoln, Y., Lynham, S., \& Guba, E. (2017). Paradigmatic controversies, contradictions, and emerging confluences, revisited. En N. Denzin, The SAGE Handbook of Qualitative Research (5ta edic., pp. 213-263). London: SAGE.

Matud, M.P. (2016). Gender and Health [en linea]. London: Intechopen. Disponible en: https://www.intechopen.com/books/gender-differences-in-different-contexts/gender-and-health

Marková, I. (2017). The making of the theory of social representations. $\mathrm{Ca}$ dernos de Pesquisa 47(163), 358-375. Recuperado de https://dx.doi.org/ $10.1590 / 198053143760$

Mendoza, M. (2015). Mujeres y sus representaciones sociales de género en el CIP Bahías de Huatulco, México. Revista de Antropología Experimental 15(3),17-35. 
Montero, A., Vergara, J., Ríos, M. y Villarroel, R. (2017). La objeción de conciencia en el debate sobre la despenalización del aborto por tres causales en Chile. Revista Chilena Obstetricia y Ginecología 82(4), 350-360.

Montero, A. \& Villarroel, R. (2018). Acritical review of conscientious objection and decriminalization of abortion in Chile. Journal of Medical Ethics 44(4), 279-283.

Montero Vega, A. y Ramírez-Pereira, M. (2020). Noción y argumentos sobre la objeción de conciencia al aborto en Chile. Revista de Bioética y Derecho 49, 59-75. Doi: 10.1344/rbd2020.49.29071

Mora, M. (2002). La teoría de las representaciones sociales de Sergei Moscovici. Athenea digital Otoño, 2, 1-25.

Santos, W., Oliveira, P., Viegas, S., Ramos, T., Policarpo, A., \& Araújo, E. (2018). Domestic Violence Against Women Perpetrated by Intimate Partner: Professionals' Social Representations in Primary Health Care. Journal of Research: Fundamental Care Online 10(3), 770-777. Doi:10.9789/21755361.2018.v10i3.770-777

Tribunal Constitucional. (2018, octubre). Sentencia del Tribunal Constitucional respecto del Requerimiento de inconstitucionalidad del artículo 13, inciso segundo, Decreto Supremo No 67, de 23 de octubre de 2018, del Ministerio de Salud, que aprueba el Reglamento para ejercer objeción de conciencia según lo dispuesto en el artículo 119 ter del Código Sanitario. Santiago, Chile. Disponible en: https://www.tribunalconstitucional.cl/ expedientes? $\mathrm{Rol}=5572$

Tuerkheimer, D. (2017) Incredible Women: Sexual Violence and The Credibility Discount University Pennsylvania Law Review 166(1), 1-57. Disponible en: https://scholarship.law.upenn.edu/penn_law_review/vol166/iss1/1/ 